\title{
Experimental Determination of Limit Adhesive Shear Stress between Solid Wall and Liquid
}

\author{
Jerzy M. Sawicki \\ Gdańsk University of Technology, Faculty of Civil and Environmental Engineering, \\ ul. G. Narutowicza 11/12, 80-233 Gdańsk, Poland, e-mail: jsaw @pg.gda.pl
}

(Received September 09, 2014; revised November 21, 2014)

\begin{abstract}
The slip boundary condition can be a very useful relation when some specific problems of hydromechanics are considered. According to the classical form of this condition, the slip of a fluid with respect to the solid wall should occur even at a very low velocity of motion. However, both theoretical analysis and certain empirical data suggest that there must be a limit value of the wall shear stress, below which the slip does not occur. According to the simplified balance of adhesive and gravitational forces, a simple experimental method for determining this stress has been proposed and applied in this paper.
\end{abstract}

Key words: hydromechanics, slip boundary condition, adhesion

\section{Notation}

The following symbols are used in this paper:

Latin letters:
$\boldsymbol{A}, A \quad-\quad$ adhesive force and its absolute value,
$D \quad-$ characteristic dimension (e.g. pipe diameter),
$F \quad-$ plate surface,
$\boldsymbol{G}, G \quad$ - gravity force and its absolute value,
$G_{W}, G_{D}-$ weight of the plate (wet and dry, respectively),
Kn $\quad-$ Knudsen number,
$L_{s} \quad-\quad$ slip length,
$n$ - - unit vector normal to the wall,
$\boldsymbol{u} \quad-$ fluid velocity,
$\boldsymbol{u}_{w}, u_{t w}-$ fluid velocity vector near the wall and its tangent component,
$\boldsymbol{v}_{w}, v_{t w}-$ wall velocity vector and its tangent component,
$w \quad-\quad$ mean fluid velocity,
$z \quad-$ coordinate normal to the wall. 
Greek letters:

$\beta-$ empirical coefficient,

$\lambda \quad-$ hydraulic loss coefficient,

$\mu \quad-$ dynamic coefficient of liquid viscosity,

$\rho \quad-$ liquid density,

$\tau_{W} \quad$ - tangential momentum accommodation coefficient,

$\tau_{C A}-$ limit adhesive shear stress,

$\tau_{S} \quad-$ wall shear stress.

\section{General Remarks}

Interfacial effects play a very important role in hydromechanics. It is useful to classify them into two categories. The first comprises processes characterized by the presence of the free surface (liquid-gas interface), in many cases together with the liquid-solid contact surface. One can find there some important problems, such as the rising of gas bubbles in liquid, the spreading of liquid drops on a horizontal solid surface, the spreading of liquid layers on tilted plates, dynamics of rivulets, dye-swell, meniscus location, capillary forces, and many others. A fair number of these effects of both cognitive and practical importance is identified and quite well described in the objective bibliography, e.g. (Davis 1983, Hansen and Toong 1971).

The second category includes diphase situations with the fluid-solid interface only. Adhesive attraction among the molecules of the two substances causes the fluid and solid surfaces to stick to each other (which may result in the formation of a rigid wall, bounding the stream, or create an outer surface of the immersed body).

For the majority of fluid-flow phenomena this sticking can be regarded as firm binding. In consequence, for such cases one can formulate the fundamental condition of the equality of fluid and solid contact velocities:

$$
\boldsymbol{u}_{w}=\boldsymbol{v}_{w} .
$$

The above statement is probably the basic boundary condition for fluid flows, which are described by differential equations.

However, there also exists another class of problems, for which Eq. (1) leads to unrealistic calculation results, as velocities on both sides of the fluid-solid interface are different in these cases (e.g. motion of fluid films, corner flows, outflow of chemically complex liquids, such as polymers, from thin tubes (Derek et al 2002, Koplik and Banavar 1995, Moffatt 1964). Also, when the walls are sufficiently smooth, the non-slip condition can fail (Granick et al 2004).

\section{Slip-Flow Boundary Condition}

The question of boundary conditions in fluid mechanics was especially intensively discussed in the 19th century. 
The possibility of slip on the fluid-solid interface was noted nearly two hundred years ago. The proper mathematical relation was formulated by the very well-known scientist Navier in 1823. Originally, it was expressed as a linear function between velocity parallel to the boundary and boundary shear stress (i.e. Hankel boundary condition) (Thompson and Troian 1997):

$$
\beta u_{t w}=\mu \frac{\partial u_{t w}}{\partial z} .
$$

The proportionality factor $\beta$ that appears in this relation can be determined on the basis of the kinetic theory of fluids. Since the boundary, in a general case, can move with the velocity $\boldsymbol{v}$, Eq. (2) can be written as the Maxwell boundary condition ( $D-$ characteristic flow dimension, e.g. pipe diameter):

$$
u_{t w}-v_{t w}=\frac{2-\sigma_{w}}{\sigma_{w}} K n D \frac{\partial u_{t w}}{\partial z} .
$$

where $\sigma_{w}$ is the tangential momentum accommodation coefficient (Shen et al 2007), and $\mathrm{Kn}$ is the Knudsen number (Puzyrewski and Sawicki 2000).

From the practical point of view, the most convenient form of this condition contains the so-called slip length $L_{s}$ (Khaled and Vafai 2004, Marques et al 2000, Zhu and Granick 2001):

$$
u_{t w}-v_{t w}=L_{s} \frac{\partial u_{t w}}{\partial z} .
$$

It is necessary to point out that these relations have a formal character, i.e. they express the boundary condition that should be satisfied when the equations of fluid motion are considered. From the physical point of view, the behavior of fluid in the vicinity of a rigid wall is a very complex question. On the one hand, one should analyze the shear stress in the fluid, described by the Newton's hypothesis, and on the other hand, chemical and physical interactions between the wall and fluid materials (Granick et al).

\section{Objection to Slip Condition}

According to the mathematical form of Eq. (4), the velocity jump caused by the slip is a continuous function of a normal derivative of a tangent velocity. This means that the fluid slip with respect to the wall must occur even for very slow motion.

But this statement is open to a serious objection. Namely, from the physical point of view, an effective slip of the fluid along the wall can occur only after the overcoming of the adhesive force, which binds the fluid and the solid together. Thus, the slip should rather be expected under more dynamic flow conditions.

This conclusion seems to be confirmed by the results of investigations of the relation between slip length and liquid velocity (Zhu and Granick 2001). As shown in 
Fig. 1, there is some minimal value of the argument $(v / D)$ for each liquid analyzed. Below this threshold, the slip does not occur (i.e. $L_{s}=0$ ).

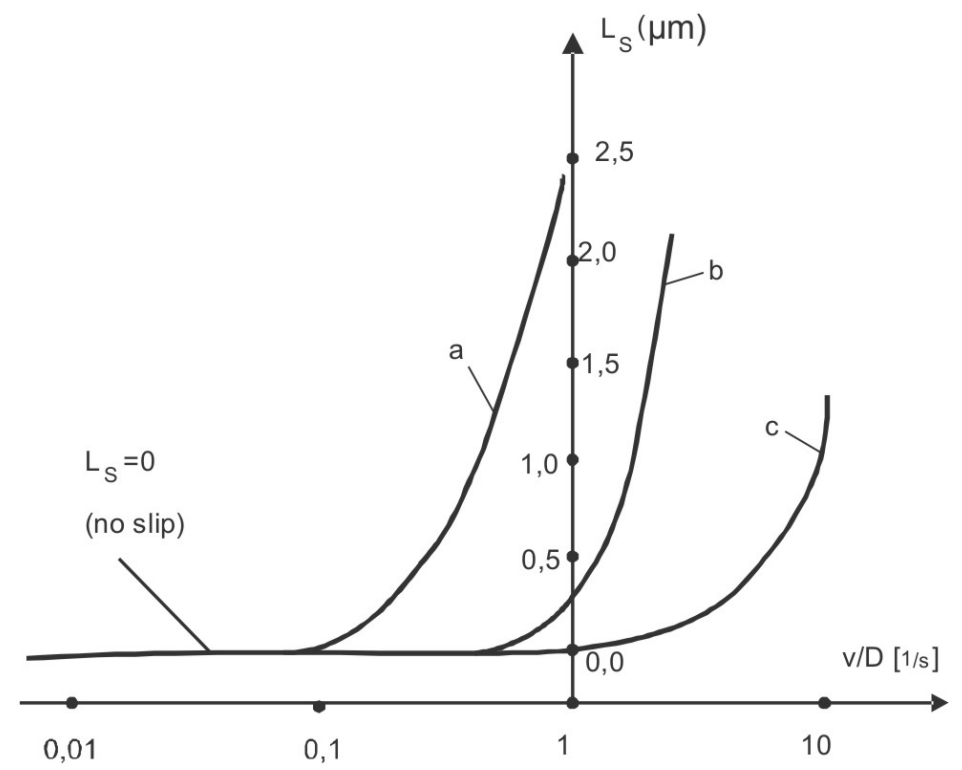

Fig. 1. The relationship between liquid velocity and slip length (a - deionized water, $\mathrm{b}$ - tetradecane, $\mathrm{c}$ - tetradecane, and $0.2 \%$ hexadecylamine)

It is worth noting that the quotient $(v / D)$ is practically equivalent to the wall shear stress in laminar flow, which can be expressed by the well-known expression (Puzyrewski and Sawicki 2000):

$$
\tau_{s}=\frac{8 \mu w}{D}
$$

\section{Concept of Limit Adhesive Shear Stress}

In conclusion, one might expect the slip to occur only when there is some minimal fluid momentum flux, perpendicular to the wall. In terms of continuous medium mechanics, one could say that the shear stress between the liquid and the wall should be strong enough to move fluid molecules adjacent to the solid surface. Its minimal value, which separates slip and non-slip flow conditions, can be called the "limit adhesive shear stress."

One could try to determine the value of this parameter by the structural approach, on the basis of the molecular theory of matter. However, for the expression thus developed/formulated to be useful in classical hydromechanics, this value should have a phenomenological character and should be determined experimentally. 
A simple model of the phenomenon under discussion is proposed here. It is based on the assumption that a solid body, submerged and then taken out of the wetting liquid (water in this case), remains wet, i.e. retains some amount of this liquid on its outer surface, which is a consequence of the adhesive force.

In the case of a thin flat plate oriented vertically (Fig. 2a), the adhesive force $A$, which keeps the adjacent liquid layer on the plate must be related (or - at least - proportional) to the gravity force $G$, which acts downwards (Fig. 2b) and tends to remove this layer from the plate surface (i.e. to cause this liquid to slip off the surface).

a)

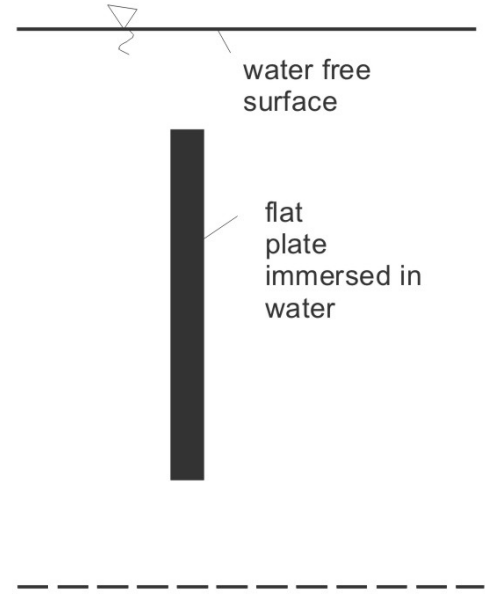

b)

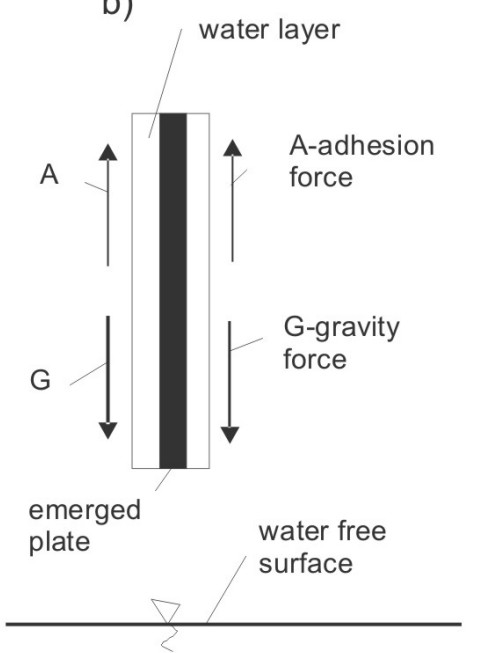

Fig. 2. Scheme for the determination of the limit adhesive shear stress

Thus, according to this concept, the determination of the weight of the adjoined liquid $G$ makes it possible to calculate the limiting adhesive shear stress $\tau_{C A}(F-$ plate surface):

$$
\tau_{C A}=\frac{A}{2 F}=\frac{G}{2 F} .
$$

Obviously, the proposed parameter is of a synthetic nature, and its precision is limited by the simplifications of the model (especially, the neglect of the plate's thickness and the influence of the surface tension). During measurements one should ensure that the wettability of the plate surface is uniform. Otherwise, the emerged plate will be covered with separate drops of water, instead of a possibly regular layer of this liquid.

\section{Laboratory Investigations}

Measurements were taken for eight different materials: glass, Plexiglass, Bakelite, aluminum, stainless steel, galvanized steel, copper, and brass. The height of each plate 
used for the measurements was $75.0 \mathrm{~mm}$, width $-50.0 \mathrm{~mm}$, and thickness $-1.0 \mathrm{~mm}$. The weight of water adhering to the emerged plate was determined according to the following procedure:

1. Setting-up and reading the water temperature in the reservoir (fed in a closed cycle through a thermostat).

2. Weighing the dry plate (value $G_{D}$ ).

3. Washing the plate in denatured alcohol (in order to remove impurities affecting its wettability).

4. Immersion of the vertically oriented plate in the water reservoir.

5. Slow emergence of the plate.

6. Weighing the wet plate (value $G_{W}$ ).

7. Calculating the weight of the adhering water:

$$
G=G_{W}-G_{D} .
$$

8. Calculating the limiting adhesive shear stress (Eq. 6).

For each plate and each temperature $\left(20^{\circ} \mathrm{C}, 30^{\circ} \mathrm{C}, 40^{\circ} \mathrm{C}\right.$, and $\left.50^{\circ} \mathrm{C}\right)$ the measurement was repeated five times, and then the mean value was determined. The results obtained are presented in Fig. 3 .

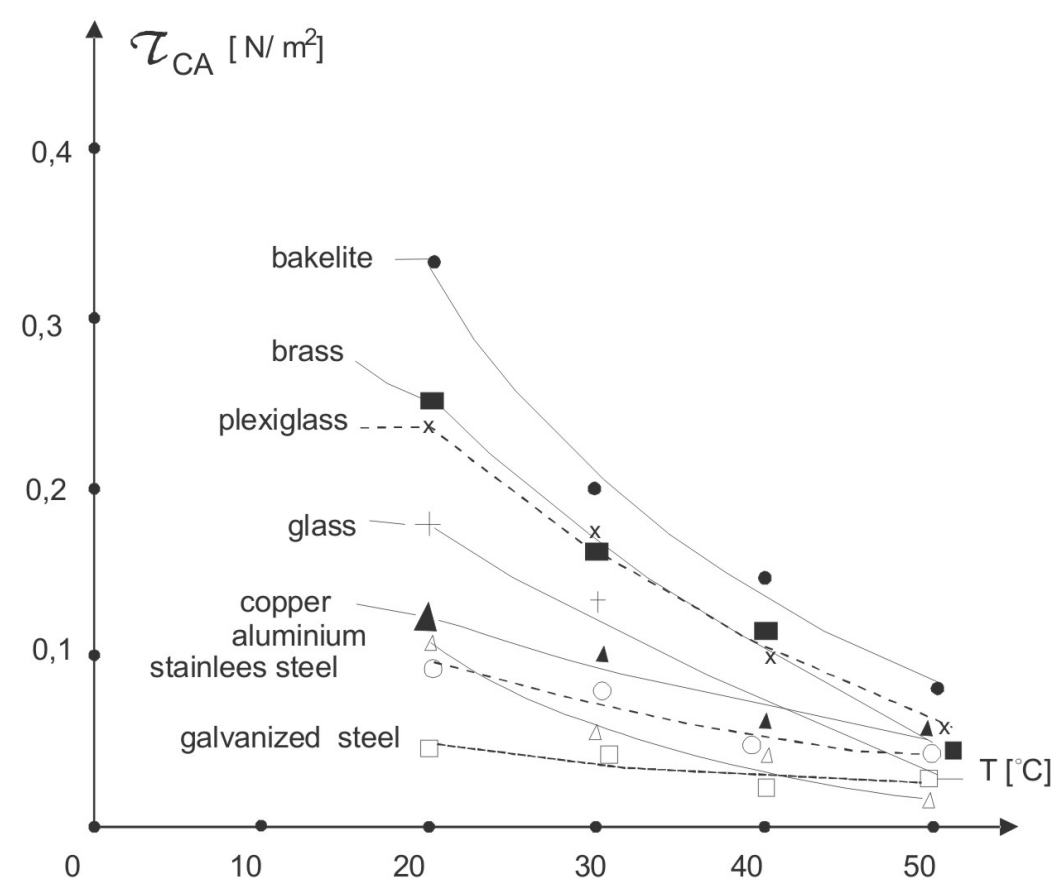

Fig. 3. Limit adhesive shear stress at different temperatures 


\section{Conclusions}

The purpose of this paper has been to determine a certain criterial value, called the limit adhesive shear stress. To some degree, it has a hypothetical character, but its introduction is justified by both theoretical analysis (comparison of two forces: adhesion and gravitation) and experimental results (Fig. 1).

The values of $\tau_{C A}$ (Fig. 3), obtained by measurements for different plate materials, show a considerable differentiation. The highest, for Bakelite, is over ten times greater than the lowest, for galvanized steel. The order of these materials in terms of the $\tau_{C A}$ value, established by the experiment, is rather surprising. Unfortunately, it did not succeed in explaining this order, so the problem will be analyzed in the course of further investigations. On the basis of the results obtained so far, one can conclude that the slip between liquid and a solid wall can be expected when the wall shear stress exceeds the limit value defined in this paper. For the pipe flow, one can write:

$$
\tau_{S}=\lambda \frac{\rho w^{2}}{8}>\tau_{C A}
$$

\section{References}

Davis S. H. (1983) Contact-line Problems in Fluid Mechanics, J. of Appl. Mech., 12, 977-982.

Derek C., Tretheway D. C., Mainhart C. D. (2002) Apparent Fluid Slip at Hydrophobic Microchannel Wall, Phys. of Fluids, 14, 9-12.

Granick S., Zhu Y., Lee H. (2004) Slippery questions about complex fluuids flowing past solids, Nature Materials, 2, 221-227.

Hansen R. J., Toong T. Y. (2001) Rate-dependent Slip of Newtonian Fluids at Smooth Surface, Phys. Rev. Letters, 9, 196-207.

Khaled A. R. A., Vafai K. (2004) The Effect of the Slip Condition on Stokes and Couette Flows Due to an Oscillatory Wall: Exact Solution, Int. J. of Non-linear Mechanics, 39, 795-809.

Koplik J., Banavar J. R. (1995) Corner Flow in the Sliding Plate Problem, Phys. of Fluids, 7, 3118-3125.

Marques R. J., Kremer G. M., Sharipov F. M. (2000) Couette Flow with Slip and Jump Boundary Condition, Continuum Mech. Thermodyn., 12, 379-386.

Moffatt H. K. (1964) Viscous and Resistive Eddies Near a Sharp Corner, J. of Fluid Mech., 18, 1-18.

Navier C. L. (1823) Memoire sur les lois des movement des fluids, Mem. Acad. Sci. Inst. France, 6.

Puzyrewski R. Sawicki J. M. (2000) Fundamentals of Fluid Mechanics and Hydraulics (in Polish), $3^{\text {rd }}$ ed., PWN, Warsaw.

Shen S., Chen G., Crone R. M., Anaya-Dufresne M. (2007) A Kinetic Theory Based on First-order Slip Boundary Condition for Gas Flow, Phys. of Fluids, 19, 18-37.

Thompson P. A., Troian S. M. (1997) A General Boundary Condition for Liquid Flow at Solid Surfaces, Nature, 389, 360-363.

Zhu Y., Granick S. (2001) Rate-Dependent Slip of Newtonian Liquids at Smooth Surfaces, Phys. Rev. Letters, 9, 1-4. 